\title{
Dynamics and Stability Issues of a Single-Inductor Dual-Switching DC-DC Converter
}

\author{
Vanessa Moreno-Font, Abdelali El Aroudi, Member, IEEE, Javier Calvente, Member, IEEE, \\ Roberto Giral, Member, IEEE, and Luis Benadero
}

\begin{abstract}
A single-inductor two-input two-output power electronic dc-dc converter can be used to regulate two generally nonsymmetric positive and negative outputs by means of a pulsewidth modulation with a double voltage feedback. This paper studies the dynamic behavior of this system. First, the operation modes and the steady-state properties of the converter are addressed, and, then, a stability analysis that includes both the power stage and control parameters is carried out. Different bifurcations are determined from the averaged model and from the discrete-time model. The Routh-Hurwitz criterion is used to obtain the stability regions of the averaged (slow-scale) dynamics in the design parameter space, and a discrete-time approach is used to obtain more accurate results and to detect possible (fast-scale) subharmonic oscillations. Experimental measurements were taken from a system prototype to confirm the analytical results and numerical simulations. Some possible nonsmooth bifurcations due to the change in the switching patterns are also illustrated.
\end{abstract}

Index Terms-Bifurcations, dc-dc converters, dual output, nonlinear dynamics, power electronics, single inductor, stability analysis.

\section{INTRODUCTION}

$\mathbf{T}$ HE BEHAVIOR of the dual-switching converter for automotive applications presented in [1] can have a rich set of instability phenomena. This system is a third-order nonisolated $\mathrm{dc}-\mathrm{dc}$ switching converter that can be derived from a noninverting buck-boost converter [2], [3]. Some of these phenomena were first studied for this system in [4] in which averaging techniques were used to detect Hopf and saddle-node bifurcations. The results provided in [4] have been validated here by means of numerical simulations.

There is an extensive literature devoted to nonlinear dynamics in elementary $\mathrm{dc}-\mathrm{dc}$ converters [5]-[12]. More recent results related to these topics can be found in [13] in which studies of the existence of bifurcations and chaotic behavior in buck converters controlled by pulsewidth modulation (PWM) of the first kind (PWM-1) have been carried out using an exact discrete-time model. $<<\mathrm{AQ} 3>>$ The PWM-1 is a variant of PWM in which the value of the control signal is sampled at the beginning of the switching cycle and compared with the ramp waveform to generate the switching signal. Further research into buck

Manuscript received July 09, 2008; revised October 21, 2008. First published June 02, 2009; current version published February 10, 2010.This work was supported by the Spanish Grants TEC-2007-67988-C02-02 and TEC2009-13172. This paper was recommended by Associate Editor M. di Bernardo.

V. Moreno-Font and L. Benadero are with the Department of Applied Physics, Universitat Politècnica de Catalunya (UPC), 08034 Barcelona, Spain.

A. El Aroudi, R. Giral, and J. Calvente are with the Department of Electronics, Electrical Engineering and Automatic Control (DEEEA), Universitat Rovira i Virgili (URV), 43007 Tarragona, Spain (e-mail: abdelali.elaroudi@urv.cat).

Digital Object Identifier 10.1109/TCSI.2009.2023769 converters under zero-average dynamics control can be found in [14].

Other techniques for analyzing stability in these systems have also been used, such as the sensitivity matrix [15], [16], the auxiliary state vector [17], and the Filippov's method [18]-[20], which give similar results to those obtained from the discretetime model. The application of nonlinear tools has not been restricted to elementary dc-dc converters: More complex systems such as the Cuk converter [21], [22] and power-factor-correction circuits have also been studied with a nonlinear approach [23]-[26].

Nonlinear analysis has also been extended to multi-input multioutput dc-dc power electronic circuits, such as multicell converters [27], [28] and multichannel converters [29], [30]. Several topologies and strategies for controlling single-inductor multiple-output (SIMO) dc-dc converters can be found in [31]-[35], which use one inductor to transfer energy from one battery to several outputs.

The main question that remains to be addressed is the problem of controlling and regulating SIMO converters. Time multiplexing is a simple and safe option [33], but to avoid cross regulation between outputs and guarantee their stability, there must be a zero inductor current during some specific time intervals. Therefore, the outputs are independently controlled, even if they share the same inductor, and the converter must operate in discontinuous conduction mode (DCM). The pseudocontinuous conduction mode (pseudo-CCM) uses a constant value for the inductor current as a virtual zero, and its operating mode is equivalent to DCM [34]. Different approaches under CCM operation and digital control with separate regulation of common and differential modes for the output voltages are outlined in [35]. The converter studied in this paper uses a single inductor that transfers energy from an unregulated source to the two loads, and two feedback loops to regulate the two output voltages by means of PWM techniques.

Studying the dynamics of the two-input two-output (TITO) $\mathrm{dc}-\mathrm{dc}$ converter in terms of the number of energy storage elements, the allowable number of configurations, and the operation modes is quite complex, and analyzing this converter's stability is a real challenge. In this paper, the stability and dynamic behavior of this system are reanalyzed to gain further insights. As the averaging methods were unable to justify some instability phenomena detected in the switched model [5]-[8], the discrete-time model was used in this study. We have also experimentally validated the most significant results presented in [4]. The system dynamics are studied from different perspectives, and appropriate approaches are used to analyze both the slow-scale (averaged) and the fast-scale (switched) dynamics. In 


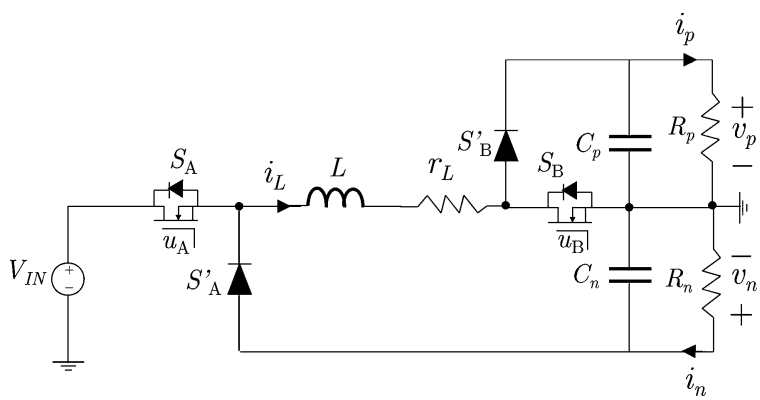

Fig. 1. TITO dc-dc switching converter.

contrast to previously published works, we observed that sometimes the averaged model fails to predict not only the period doubling (PD) bifurcation but also the Hopf bifurcation. Therefore, a discrete formulation is introduced in order to study the system's stability taking into account the switching action. We found that this model is able to predict a variety of instabilities that can occur in the system.

This paper is organized as follows: In Section II, the circuit is described, and its switched model is introduced. In Section III, the experimental system is described. In Section IV, the main characteristic relations between averaged state variables and duty cycles are given. An explicit expression for the Jacobian matrix of the averaged model and some representative results for stability are derived in the same section. In Section $\mathrm{V}$, extensive numerical simulations are carried out to determine most of the dynamic behaviors that the system can show, and the shortcomings of the averaging approach are highlighted. In Section VI, discrete-time formulation is used to accurately determine the stability boundary in a suitable parameter space. In Section VII, specific bifurcations due to discontinuities in the power stage or in the control are presented. Experimental measurements were also taken to confirm our analytical results and numerical simulations. Finally, some conclusions are presented in the last section.

\section{SYSTEM DESCRIPTION}

\section{A. Power Stage Description}

The single-inductor dual-switching dc-dc power converter scheme is shown in Fig. 1 and is composed of the following elements: the unregulated power source voltage $V_{I N}$; the inductor modeled by its equivalent series circuit inductance $L$ and resistance $r_{L}$; the positive-channel load resistance $R_{P}$ and filter capacitance $C_{P}$; and the negative-channel load $R_{N}$ and filter capacitance $C_{N}$. Two ideal switches $S_{A}$ and $S_{B}$ are also included: $S_{A}$ connects the inductor either to the main source (ON case) or to the negative load (OFF case); $S_{B}$ connects the other terminal of the inductor either to the ground (ON case) or to the positive channel load (OFF case). This converter has three state variables associated with the energy storage elements, the inductor and the filter capacitors: the inductor current $i_{L}$ and the capacitor voltages $v_{P}$ and $v_{N}$ corresponding to the positive and negative channels, respectively.

The converter operates by switching among different topologies. In the CCM, the inductor current is always defined as positive, and thus, the action of the diode is complementary to the

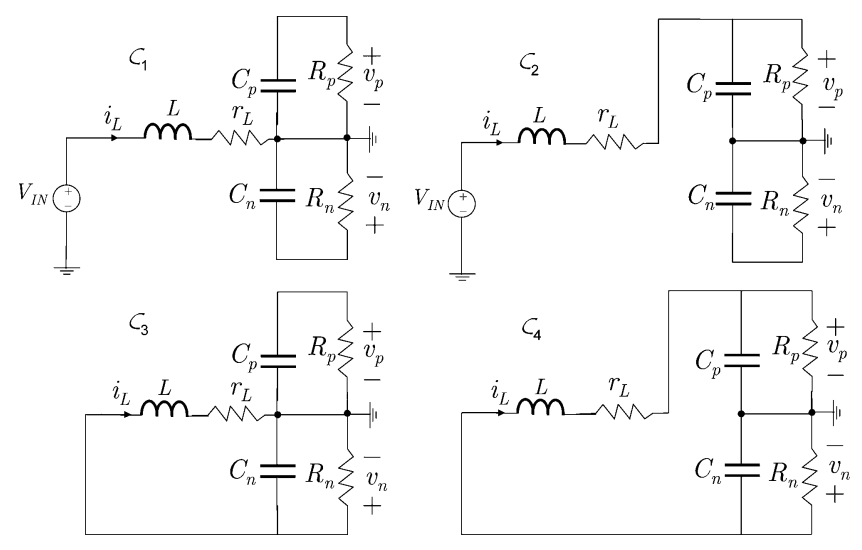

Fig. 2. Possible configurations of the TITO dc-dc switching converter.

corresponding controlled switch. As there are two independent switches, at least four topologies are possible.

Both the $S_{A}$ and $S_{B}$ switches are implemented by means of a MOSFET (controlled switch) and a diode (uncontrolled switch) (Fig. 1). One configuration, called $\mathcal{C}_{1}$ : (ON, ON), is given when both controlled switches are ON (so, the diodes are open), and the source transfers energy to the inductor by increasing its current $i_{L}$. Another configuration, called $\mathcal{C}_{2}$ : (ON,OFF), is given when switch $S_{B}$ changes to the OFF state while $S_{A}$ remains ON, and the inductor (still connected to the source) transfers energy only to the positive load. Note that if the switching sequence $\mathcal{C}_{1} \rightarrow \mathcal{C}_{2}$ runs (so $S_{A}$ is always ON), the circuit will in fact operate as a boost converter $\left(v_{n}=0\right)$.

The configuration $\mathcal{C}_{3}$ : (OFF, ON) appears if switch $S_{A}$ changes to OFF while $S_{B}$ remains ON, so the inductor (now disconnected from the source) transfers energy only to the negative load. As in the previous case, if the sequence $\mathcal{C}_{1} \rightarrow \mathcal{C}_{3}$ occurs $\left(S_{B}\right.$ being always ON), the circuit will operate as a buck-boost switching converter $\left(v_{p}=0\right)$.

In the fourth configuration $\mathcal{C}_{4}:(\mathrm{OFF}, \mathrm{OFF})$, when both switches are open, the energy flows from the inductor to both the positive and negative channel loads; therefore, this topology corresponds to a series connection of the loads and the inductor. A variant of this configuration occurs if, during some time interval, the inductor current drops to zero because all transistors and diodes are open. In this case, the converter is said to operate in DCM. This mode is taken into account in the simulations but not in the analysis, in which the number of operating topologies has been limited to the four described earlier (see Fig. 2).

Except in the particular symmetrical case with equal loads $\left(R_{N}=R_{P}\right)$ and equal absolute values of the output voltages $\left(v_{N}=-v_{P}\right)$, for which both switching actions are simultaneous, independent switching is necessary due to unbalanced loads or different absolute voltage output requirements. Duty ratios $d_{A}$ and $d_{B}$ will therefore be different.

\section{B. Continuous-Conduction Operating Modes}

There are different operating modes for the system depending on the relationship between the duty cycles of the driving signals, switching frequency, value of the inductor, and loads. However, it can be shown that, assuming the inductor 

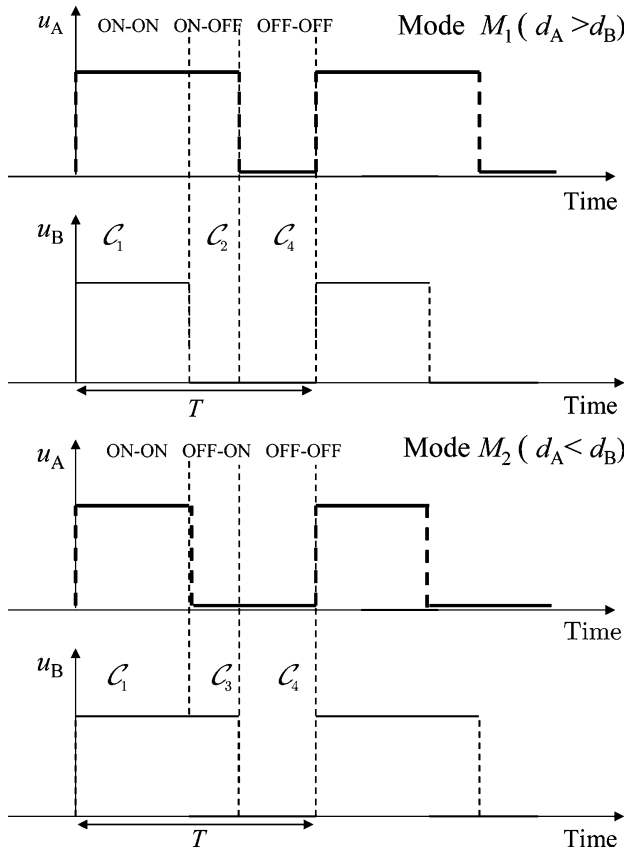

Fig. 3. Continuous-conduction operating modes for the TITO dc-dc converter.

current never drops to zero, there are basically two operating modes, which can be summarized as follows (Fig. 3).

1) Mode $\boldsymbol{M}_{1}$ : The switching sequence is $\mathcal{C}_{1} \rightarrow \mathcal{C}_{2} \rightarrow \mathcal{C}_{4}$. This mode appears for $d_{A}>d_{B}$.

2) Mode $\boldsymbol{M}_{2}$ : The switching sequence is $\mathcal{C}_{1} \rightarrow \mathcal{C}_{3} \rightarrow \mathcal{C}_{4}$. This mode appears for $d_{A}<d_{B}$.

Roughly speaking, $d_{A}>d_{B}$ and $d_{A}<d_{B}$ favor the positive and negative current load, respectively.

If the time duration for each topology are called $\boldsymbol{t}_{\mathcal{C}_{1}}, \boldsymbol{t}_{\mathcal{C}_{2}}, \boldsymbol{t}_{\mathcal{C}_{3}}$, $\boldsymbol{t}_{\mathcal{C}_{4}}$, and the switching period is called $T$, the duty ratio definitions for the two cases are as follows:

Mode $M_{1}, \mathcal{C}_{3}$ skipped: $d_{A}=\frac{t_{\mathcal{C}_{1}}+t_{\mathcal{C}_{2}}}{T}, \quad d_{B}=\frac{t_{\mathcal{C}_{1}}}{T}$

Mode $M_{2}, \mathcal{C}_{2}$ skipped: $d_{A}=\frac{t_{\mathcal{C}_{1}}}{T}, \quad d_{B}=\frac{t_{\mathcal{C}_{1}}+t_{\mathcal{C}_{3}}}{T}$

\section{Switched Model of the Open-Loop System}

The switched model of a dc-dc converter is a convenient tool for simulating the converter's dynamics. As every topology is linear, these systems are classified as piecewise linear, and their dynamics are straightforward to determine from the corresponding linear differential equation and initial condition sets.

For each configuration $\mathcal{C}_{k}(k \in\{1,2,3,4\})$ of the switching system, the state equations are linear time invariant in the form of $\dot{\mathbf{x}}=\mathbf{A}_{k} \mathbf{x}+\mathbf{B}_{k}$, where $\mathbf{x}=\left(\begin{array}{lll}v_{p} & v_{n} & i_{L}\end{array}\right)^{\mathrm{T}}$ is the vector of the state variables for the power stage circuit, $\mathbf{A}_{k}$ and $\mathbf{B}_{k}$ are the system matrix and input vector, respectively, during phase $k$, and the overdot stands for derivation with respect to time.
The expressions of the state matrices and input vectors can be obtained by applying Kirchhoff's voltage law to the configurations in Fig. 2. Matrices A and $\mathbf{B}$ for each case are as follows.

1) Configuration $\mathcal{C}_{1},(\mathrm{ON}, \mathrm{ON})$

$$
\mathbf{A}_{1}=\left(\begin{array}{ccc}
\frac{-1}{R_{P} C_{P}} & 0 & 0 \\
0 & \frac{-1}{R_{N} C_{N}} & 0 \\
0 & 0 & \frac{-r_{L}}{L}
\end{array}\right), \quad \mathbf{B}_{1}=\left(\begin{array}{c}
0 \\
0 \\
\frac{V_{i n}}{L}
\end{array}\right) .
$$

2) Configuration $\mathcal{C}_{2},(\mathrm{ON}, \mathrm{OFF})$

$$
\mathbf{A}_{2}=\left(\begin{array}{ccc}
\frac{-1}{R_{P} C_{P}} & 0 & \frac{1}{C_{P}} \\
0 & \frac{-1}{R_{N} C_{N}} & 0 \\
-\frac{1}{L} & 0 & \frac{-r_{L}}{L}
\end{array}\right), \quad \mathbf{B}_{2}=\left(\begin{array}{c}
0 \\
0 \\
\frac{V_{i n}}{L}
\end{array}\right)
$$

3) Configuration $\mathcal{C}_{3},(\mathrm{OFF}, \mathrm{ON})$

$$
\mathbf{A}_{3}=\left(\begin{array}{ccc}
\frac{-1}{R_{P} C_{P}} & 0 & 0 \\
0 & \frac{-1}{R_{N} C_{N}} & \frac{-1}{C_{N}} \\
0 & \frac{1}{L} & \frac{-r_{L}}{L}
\end{array}\right), \quad \mathbf{B}_{3}=\left(\begin{array}{l}
0 \\
0 \\
0
\end{array}\right) .
$$

4) Configuration $\mathcal{C}_{4}$, (OFF, OFF)

$$
\mathbf{A}_{4}=\left(\begin{array}{ccc}
\frac{-1}{R_{P} C_{P}} & 0 & \frac{1}{C_{P}} \\
0 & \frac{-1}{R_{N} C_{N}} & \frac{-1}{C_{N}} \\
\frac{-1}{L} & \frac{1}{L} & \frac{-r_{L}}{L}
\end{array}\right), \quad \mathbf{B}_{4}=\left(\begin{array}{l}
0 \\
0 \\
0
\end{array}\right) .
$$

Alternatively, the state equation can be expressed compactly in terms of $u_{A}$ and $u_{B}$ binary signals $\left(u_{X} \in\{0,1\}, X \in\right.$ $\{A, B\})$ as follows:

$$
\dot{\mathbf{x}}=\left(\begin{array}{ccc}
\frac{-1}{R_{P} C_{P}} & 0 & \frac{1-u_{B}}{C_{P}} \\
0 & \frac{-1}{R_{N} C_{N}} & \frac{u_{A}-1}{C_{N}} \\
\frac{u_{B}-1}{L} & \frac{1-u_{A}}{L} & \frac{-r_{L}}{L}
\end{array}\right) \mathbf{X}+\left(\begin{array}{c}
0 \\
0 \\
\frac{u_{A} V_{i n}}{L}
\end{array}\right) .
$$

\section{TITO PWM Controller With Proportional Plus Integral Feedback Voltage Loop}

The objective of the control (Fig. 4) is to regulate both voltage outputs at the reference values $V_{P}$ and $V_{N}$ (positive and negative) by driving the switches so that the duty ratios are adapted to the presence of possible disturbances at the source or in the loads. Therefore, a double feedback loop must be designed to provide the binary control signals $u_{A}(t)$ and $u_{B}(t)$. The instants at which transitions between topologies occur can be determined by solving the switching equations that are defined next. A proportional-integral (PI) compensator for each output is used in order to obtain zero steady-state errors for both outputs. The use of this dynamic controller adds two extra state variables $\zeta_{P}$ and $\zeta_{N}$ to the system. The state equations for these two extra variables are the following:

$$
\begin{aligned}
\dot{\zeta}_{P}(t) & =\frac{v_{P}-V_{P}}{\tau_{P}} \\
\dot{\zeta}_{N}(t) & =\frac{V_{N}-v_{N}}{\tau_{N}} .
\end{aligned}
$$




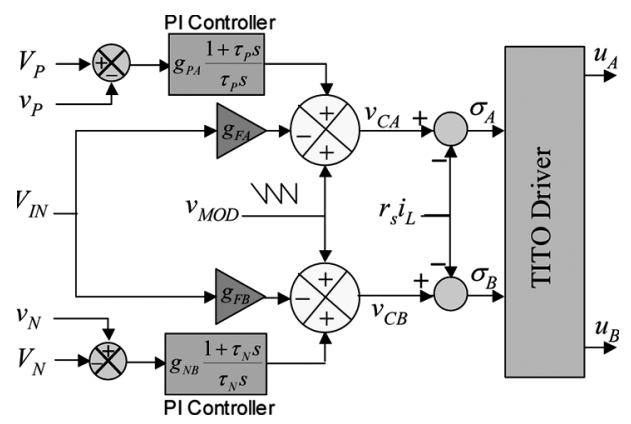

Fig. 4. Block diagram of both feedback loops for the TITO dc-dc converter.

To complete the model, the evolution of the integral variables $\zeta_{P}$ and $\zeta_{N}$ must be considered with PI control for both loops. In this case, the equations for the two switching conditions $\sigma_{A}(t)=0$ and $\sigma_{B}(t)=0$ can be written as

$$
\begin{aligned}
\sigma_{A} & =v_{C A}(t)-r_{S} i_{L} \\
& =v_{\mathrm{MOD}}(t)-g_{P A}\left(v_{P}-V_{P}+\zeta_{P}\right)-g_{F A} V_{I N}-r_{S} i_{L} \\
& =v_{\mathrm{MOD}}(t)+G_{X A}\left(\mathbf{X}_{r e f, A}-\mathbf{x}\right)-g_{F A} V_{I N}=0 \\
\sigma_{B} & =v_{C B}(t)-r_{S} i_{L} \\
& =v_{\mathrm{MOD}}(t)-g_{N B}\left(V_{N}-v_{N}+\zeta_{N}\right)-g_{F B} V_{I N}-r_{S} i_{L} \\
& =v_{\mathrm{MOD}}(t)+G_{X B}\left(\mathbf{X}_{r e f, B}-\mathbf{x}\right)-g_{F B} V_{I N}=0
\end{aligned}
$$

where $\tau_{P}$ and $\tau_{N}$ are the time constants of the integrators, $g_{P A}$ and $g_{N B}$ are the proportional gains of the PI blocks, and $r_{S}$ is the current-sensor gain. Without changing the notation, $\mathbf{x}=\left(\begin{array}{llllll}v_{P} & v_{N} & i_{L} & \zeta_{P} & \zeta_{N}\end{array}\right)^{\mathrm{T}}$ is the vector of the state variables augmented by the integral of the error voltages $\zeta_{p}$ and $\zeta_{n} \cdot \mathbf{G}_{X A}=\left(\begin{array}{lllll}g_{P A} & 0 & r_{S} & g_{P A} & 0\end{array}\right)$ and $\mathbf{G}_{X B}=\left(\begin{array}{lllll}0 & -g_{N B} & r_{S} & 0 & g_{N B}\end{array}\right)$ are the vectors of the feedback coefficients including these integral terms. $X_{\mathrm{ref}, A}=$ $\left(\begin{array}{lllll}V_{P} & 0 & 0 & 0 & 0\end{array}\right)^{\mathrm{T}}$ and $X_{\mathrm{ref}, B}=\left(\begin{array}{lllll}0 & -V_{N} & 0 & 0 & 0\end{array}\right)^{\mathrm{T}}$ are the reference vectors for the two outputs of the augmented system.

The driving signal $u_{A}(t)$ (or $u_{B}(t)$ ) is generated by comparing the control signal $v_{C A}(t)$ (or $v_{C B}(t)$ ), which includes the external modulating signal $v_{\mathrm{MOD}}(t)$ with the sensed inductor current. This modulating signal is a repetitive sawtooth function with extreme voltage values $V_{U}$ (upper) and $V_{L}$ (lower), and its expression can be written as follows:

$$
v_{\mathrm{MOD}}(t)=V_{U}-\left(V_{U}-V_{L}\right) \frac{t}{T} \bmod 1 .
$$

With the integral terms included, this system is characterized by a set of five first-order ordinary differential equations, and, therefore, it is a 5-D periodically forced system.

\section{EXPERIMENTAL PROTOTYPE OF THE TITO CONVERTER}

A prototype of a TITO dc-dc converter was built in order to confirm our analytical results and numerical simulations. The dynamic ranges of some parameters were selected according to our technical capabilities, but the results can be rescaled, for instance, to higher switching frequencies, unless parasitic component effects become relevant. The nominal values of the parameters are shown in Table I. The inductor current is sensed using a five-turn loop with the current probe PR30 to provide a total

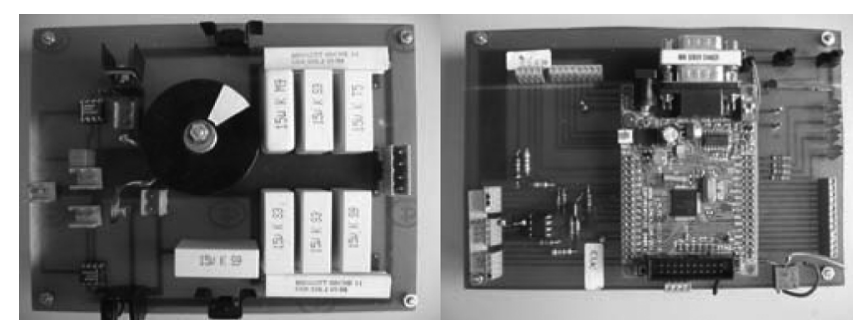

Fig. 5. Experimental prototype of a TITO dc-dc converter. Left: Power stage circuit. Right: TITO controller.

TABLE I

Nominal VAlues of The PARAMETERS USED.

\begin{tabular}{|c|c|c|c|}
\hline Parameter & Value & Parameter & Value \\
\hline Input voltage $V_{I N}$ & $6 \mathrm{~V}$ & Current sensor gain $r_{S}$ & $0.05-1 \Omega$ \\
\hline Positive reference $V_{P}$ & $12 \mathrm{~V}$ & $\begin{array}{c}\text { Proportional gains } \\
g_{P A}=g_{N B}=g\end{array}$ & $0.001-0.2$ \\
\hline Negative reference $V_{N}$ & $-5 \mathrm{~V}$ & $\begin{array}{c}\text { Time constants of the } \\
\text { integrators } \tau=\tau_{P}=\tau_{N}\end{array}$ & $25-250 \mu \mathrm{s}$ \\
\hline $\begin{array}{c}\text { Positive channel } \\
\text { load resistance } R_{P}\end{array}$ & $33 \Omega$ & $\begin{array}{c}\text { Lower value of the } \\
\text { ramp voltage } V_{L}\end{array}$ & $-2-0 \mathrm{~V}$ \\
\hline $\begin{array}{c}\text { Negative channel } \\
\text { load resistance } R_{N}\end{array}$ & $33 \Omega$ & $\begin{array}{c}\text { Upper value of the } \\
\text { ramp voltage } V_{U}\end{array}$ & $1-2 \mathrm{~V}$ \\
\hline $\begin{array}{c}\text { Positive channel } \\
\text { capacitor } C_{P}\end{array}$ & $45 \mu \mathrm{F}$ & $\begin{array}{c}\text { Switching frequency } \\
f_{S}=1 / T\end{array}$ & $10 \mathrm{kHz}$ \\
\hline $\begin{array}{c}\text { Negative channel } \\
\text { capacitor } C_{N}\end{array}$ & $45 \mu \mathrm{F}$ & $\begin{array}{c}\text { Equivalent series } \\
\text { resistance (inductor) } r_{L}\end{array}$ & $0.15 \Omega$ \\
\hline \begin{tabular}{c} 
Inductor $L$ \\
\hline
\end{tabular} & $620 \mu \mathrm{H}$ & \multicolumn{2}{|c}{} \\
\hline
\end{tabular}

gain of 0.5 V/A. The control signals are obtained and processed by a microcontroller. The feedback gains $g_{P A}, g_{N B}, \tau_{P}$, and $\tau_{N}$ are considered as design parameters that should be adjusted to obtain stable behavior. These parameters are tuned by a specific program that is loaded into the microcontroller memory. This device provides the command signals which are buffered by two dedicated MOS drivers from MAXIM (MAX626), and then applied to the MOSFET IRF9Z34S (P-channel) and IRL530N (N-channel). The diodes $S_{A}^{\prime}$ and $S_{B}^{\prime}$ are two Schottky barrier diodes (6CWQ04FN). The inductor current $i_{L}$ is represented with a scale factor of $0.5 \mathrm{~V} / \mathrm{A}$. In Fig. 5, there is a picture of the implemented circuit, which shows both the power stage circuit and the TITO PWM controller.

\section{STEADY-STATE Relations AND STABILITY BOUNDARY FROM THE AVERAGED MODEL}

This section is a first step toward analyzing the dynamic behavior of the system and its stability. As a first approach, the standard averaging technique is used to obtain a model that describes the low-frequency behavior of the system. Although, as will be seen later, this approach is not able to predict some of the dynamic behaviors of the system, useful information about the steady-state operation and the stability analysis of the corresponding equilibrium point (in the framework of averaging) can be obtained.

\section{A. Averaged Model}

If the period of the modulating signal is small enough compared with the time constants of the circuit (high-frequency modulation), the dynamics of the system can be approximated by the smooth averaged-model dynamics, in which the 
high-frequency ripple due to switching action is suppressed. The averaged model, which is equivalent to the weighted sum of the different equations describing the dynamic behavior during each phase, takes into account the time interval during which every topology is active. Consequently, this model is obtained by substituting the binary signals $u_{A}$ and $u_{B}$ in (5) by their respective duty ratios $d_{A}$ and $d_{B}$. Thus, taking into account the extra variables due to the integral terms in the feedback loop, the following 5-D averaged model is obtained:

$$
\dot{\overline{\mathbf{x}}}=\left(\begin{array}{ccccc}
\frac{-1}{R_{P} C_{P}} & 0 & \frac{1-d_{B}}{C_{P}} & 0 & 0 \\
0 & \frac{-1}{R_{N} C_{N}} & \frac{d_{A}-1}{C_{N}} & 0 & 0 \\
\frac{d_{B}-1}{L} & \frac{1-d_{A}}{L} & \frac{-r_{L}}{L} & 0 & 0 \\
\frac{1}{\tau_{P}} & 0 & 0 & 0 & 0 \\
0 & -\frac{1}{\tau_{N}} & 0 & 0 & 0
\end{array}\right) \overline{\mathbf{x}}+\left(\begin{array}{c}
0 \\
0 \\
\frac{V_{I N} d_{A}}{L} \\
-\frac{V_{P}}{\tau_{P}} \\
\frac{V_{N}}{\tau_{N}}
\end{array}\right)
$$

where $\overline{\mathbf{x}}=\left(\begin{array}{lllll}\bar{v}_{P} & \bar{v}_{N} & \bar{i}_{L} & \bar{\zeta}_{P} & \bar{\zeta}_{N}\end{array}\right)^{T}$ and the overbar stands for averaging during one switching period. The same result is obtained in [1] with the time-averaged equivalent circuit model. It can be shown that, by assuming that the control voltages vary slowly during a switching period, the following expressions for $d_{A}$ and $d_{B}$ are obtained:

$$
\begin{aligned}
d_{A} & =\frac{V_{U}-G_{X A}\left(\mathbf{x}-\mathbf{X}_{\mathrm{ref}, A}\right)}{V_{U}-V_{L}} \\
d_{B} & =\frac{V_{U}-G_{X B}\left(\mathbf{x}-\mathbf{X}_{\mathrm{ref}, B}\right)}{V_{U}-V_{L}} .
\end{aligned}
$$

The set of first-order differential equations of the open-loop circuit (9) and the expressions for the PWM duty ratios (10) define the closed-loop averaged system.

Unlike the switched model, the averaged model equations are smooth (provided there is no saturation in duty cycles), and because the explicit time dependence is suppressed, the averaged model is a 5-D autonomous system. However, it should be noted that this model is not valid beyond the control limits or in the saturation region. In these cases, $d_{X}$ is forced to be zero or one; therefore, some topology will remain active during the entire switching period.

\section{B. Steady-State Expressions}

The equilibrium point, which is the steady state for the averaged model, is obtained by setting the vector field (9) equal to zero. For an open-loop control, the pair of coordinates $\left(d_{A}, d_{B}\right)$ can be chosen freely in the range $[0,1]$. Consequently, the set of all possible steady-state vectors $\mathbf{x}$ will lie on a surface inside the 3-D state space (Fig. 6).

The fourth and fifth equations in (9) impose that at the steady state $\bar{v}_{P}=V_{P}, \bar{v}_{N}=V_{N}$ and from the first and second equations in (9), the expressions for the two duty ratios $D_{A}$ and $D_{B}$ at the steady-state operation, can be written as follows:

$$
\begin{aligned}
& D_{A}=1+\frac{V_{N}}{R_{N} I_{L}}=1+\frac{I_{N}}{I_{L}} \\
& D_{B}=1-\frac{V_{P}}{R_{P} I_{L}}=1-\frac{I_{P}}{I_{L}}
\end{aligned}
$$

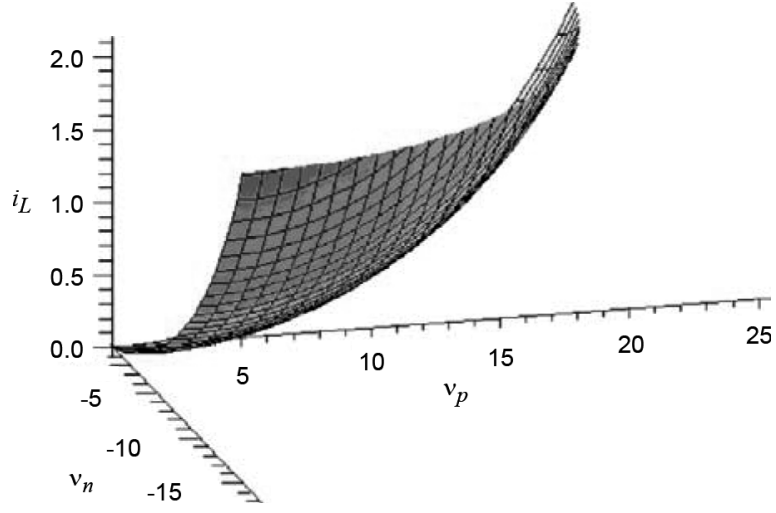

Fig. 6. Possible equilibrium point lies on a surface, which is characteristic of the TITO converter.

where $I_{N}$ and $I_{P}$ are the steady-state current loads $R_{N}$ and $R_{P}$, respectively.

Finally, an explicit expression of the inductor current in terms of the two capacitor voltages can also be obtained from the third equation in (9) together with (11), i.e.,

$$
\begin{aligned}
& I_{L}\left(V_{P}, V_{N}\right)=\frac{V_{I N}}{2 r_{L}} \\
& \mp \sqrt{\left(\frac{V_{I N}}{2 r_{L}}\right)^{2}-\frac{1}{r_{L}}\left(\frac{V_{P}^{2}}{R_{P}}+\frac{V_{N}^{2}}{R_{N}}-\frac{V_{I N} V_{N}}{R_{N}}\right) .}
\end{aligned}
$$

Expression (12) corresponds to an ellipsoid with low- and high-current regions depending on the sign of the square root; therefore, the boundary between them is a part of an ellipse obtained when the square root in (12) is zero, i.e.,

$$
I_{L}=\frac{V_{I N}}{2 r_{L}} \Rightarrow \frac{V_{P}^{2}}{R_{P}}+\frac{V_{N}^{2}}{R_{N}}-\frac{V_{I N} V_{N}}{R_{N}}=\frac{V_{I N}^{2}}{4 r_{L}}
$$

Although in (12) both positive and negative signs are possible, only the negative sign corresponds to a practical design because the positive case implies high inductor current and, therefore, high losses in the equivalent series resistance of the inductor. Note that if either $v_{P}=0$ (so $d_{B}=1$ ) or $v_{N}=0$ (so $d_{A}=1$ ), the circuit will degenerate in either the buck-boost or the boost converter, respectively. Indeed, if $r_{L}$ is neglected, (12) tends to the well-known parabolic approximations for these elementary dc-dc converters.

Alternatively, (12) can be deduced from the energy balance equation

$$
V_{I N} D_{A} I_{L}=r_{L} I_{L}^{2}+\frac{V_{P}^{2}}{R_{P}}+\frac{V_{N}^{2}}{R_{N}}
$$

Fig. 7 shows the waveforms of the system starting from zero initial conditions. The results are obtained from the direct simulation of both the averaged and the switched models. Note that in the case of a PI-controlled system, the steady-state averaged values of both voltages, 12 and $-5 \mathrm{~V}$ are imposed by the voltage references $V_{P}$ and $V_{N}$. The averaged steady-state inductor current $(\cong 1.1 \mathrm{~A})$ agrees well with (12). Note also that the slow dynamics is described well by the averaged model. 

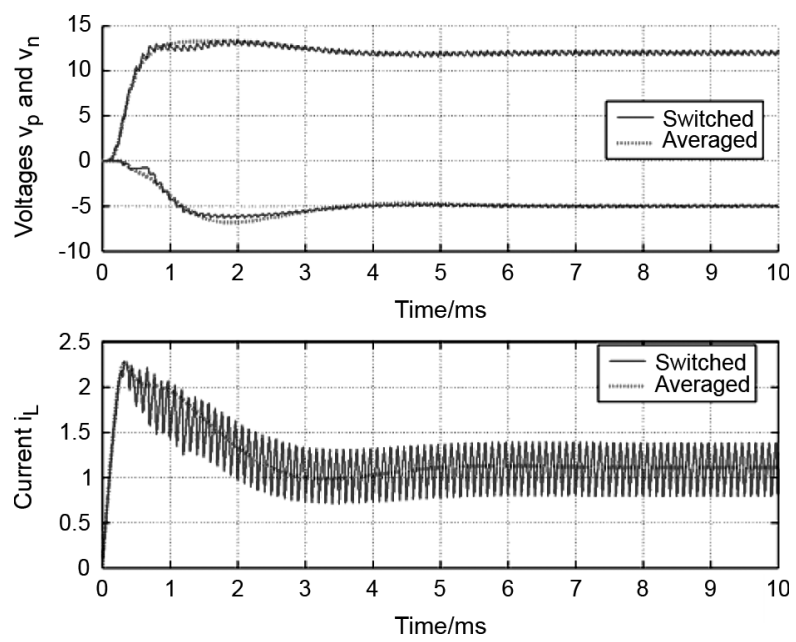

Fig. 7. Transient and steady-state waveforms of the TITO dc-dc converter from numerical simulations of the switched and averaged models. The parameter values used are as follows: $g_{P A}=g_{N B}=0.1, V_{L}=0 \mathrm{~V}, V_{U}=1.5 \mathrm{~V}$, $\tau_{P}=\tau_{N}=250 \mu \mathrm{s}$, and $r_{S}=1 \Omega$. The other parameters are from Table I.

Fig. 8 shows the steady-state behavior in the normal periodic operating mode, which also shows good agreement between the simulation results and experimental measurements.

In the next section, the averaged model is used to obtain some insights into the dynamic behavior and steady-state relations of the averaged dynamics.

\section{Stability Analysis by Using the Averaged Model}

The behavior of a dynamic system in the vicinity of its equilibrium points is topologically equivalent to the linearized system at these points [37]. Thus, the stability of the averaged dynamics of our system can be investigated from the properties of the corresponding Jacobian matrix calculated at the equilibrium points. The generic $\mathbf{J}_{i j}$ element of the Jacobian matrix is obtained as the first derivative of the $i$ th field equation in (9) with respect to the $j$ th element of the state vector $\mathbf{x}$. Evaluated at the equilibrium points, the Jacobian matrix is

$$
\mathbf{J}=\left(\begin{array}{ccccc} 
& & & 0 & \frac{g_{N B} I_{L}}{V_{U}-V_{L}} \frac{1}{C_{P}} \\
& \mathbf{J}_{P} & & \frac{-g_{P A} I_{L}}{V_{U}-V_{L}} \frac{1}{C_{N}} & 0 \\
& & & -\frac{g_{P A} V^{\prime}}{V_{U}-V_{L}} \frac{1}{L} & -\frac{g_{N B} V_{P}}{V_{U}-V_{L}} \frac{1}{L} \\
\frac{1}{\tau_{P}} & 0 & 0 & 0 & 0 \\
0 & -\frac{1}{\tau_{N}} & 0 & 0 & 0
\end{array}\right)
$$

where block $\mathbf{J}_{p}$ is given by the equation shown at the bottom of the page, with $D_{A}$ and $D_{B}$ as the duty ratios and $I_{L}$ as the inductor current that can be obtained from (11) and (12). $1-D_{A}$ is abbreviated as $D_{A}^{\prime}, 1-D_{B}$ as $D_{B}^{\prime}$, and $V^{\prime}=V_{I N}-V_{N}$, as shown at the bottom of the page.
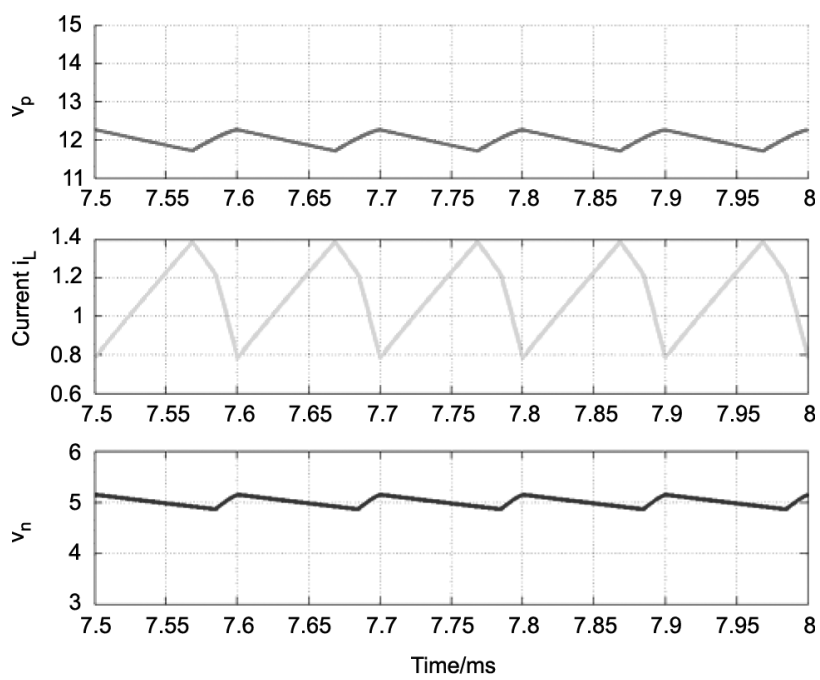

CH1:2.000V/DIV DC CH2:2.000V/DIV DC CH3: 0500V/DIV DC TB A:50 $\mu \mathrm{s}$

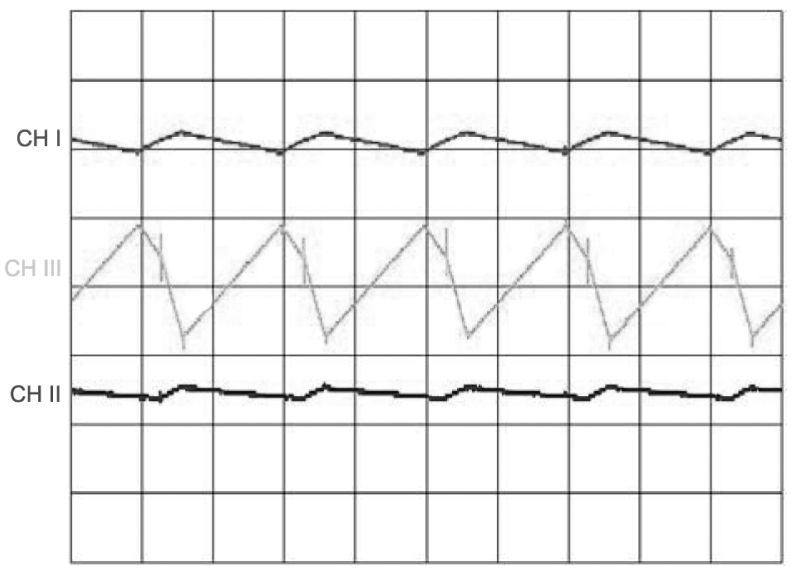

Fig. 8. Steady-state response of the TITO dc-dc converter in the normal periodic behavior derived from (top) numerical simulations and (bottom) experimental measurements. The parameter values used are $g_{P A}=g_{N B}=0.1$, $V_{L}=0 \mathrm{~V}, V_{U}=1.5 \mathrm{~V}, \tau_{P}=\tau_{N}=250 \mu \mathrm{s}$, and $r_{S}=1 \Omega$. The other parameters are from Table I.

The characteristic polynomial equation of this Jacobian matrix is a fifth-order system that can be written in the following form:

$$
\begin{aligned}
p_{5}(\lambda) & :=\operatorname{det}\left(\mathbf{J}_{P}-s \mathbf{I}\right) \\
& =s^{5}+a_{4} s^{4}+a_{3} s^{3}+a_{2} s^{2}+a_{1} s+a_{0}
\end{aligned}
$$

where $\mathbf{I}$ is the identity matrix with appropriate dimension. The coefficients $a_{k}$ depend on all the parameters of the system. The Routh-Hurwitz criterion is used in order to obtain the stability regions of the averaged dynamics. The main design parameters considered here are the parameters of the external voltage loops.

$$
\mathbf{J}_{P}=\left(\begin{array}{ccc}
-\frac{1}{R_{P} C_{P}} & -\frac{g_{N B} I_{L}}{V_{U}-V_{L}} \frac{1}{C_{P}} & \left(D_{B}^{\prime}+\frac{r_{S} I_{L}}{V_{U}-V_{L}}\right) \frac{1}{C_{P}} \\
-\frac{g_{P A} I_{L}}{V_{U}-V_{L}} \frac{1}{C_{N}} & -\frac{1}{R_{N} C_{N}} & -\left(D_{A}^{\prime}+\frac{r_{S} I_{L}}{V_{U}-V_{L}}\right) \frac{1}{C_{N}} \\
-\left(D_{B}^{\prime}+\frac{g_{P A} V^{\prime}}{V_{U}-V_{L}}\right) \frac{1}{L} & \left(D_{A}^{\prime}+\frac{g_{N B} V_{P}}{V_{U}-V_{L}}\right) \frac{1}{L} & -\left(r_{L}+\frac{r_{S}\left(V_{P}+V^{\prime}\right)}{V_{U}-V_{L}}\right) \frac{1}{L}
\end{array}\right)
$$




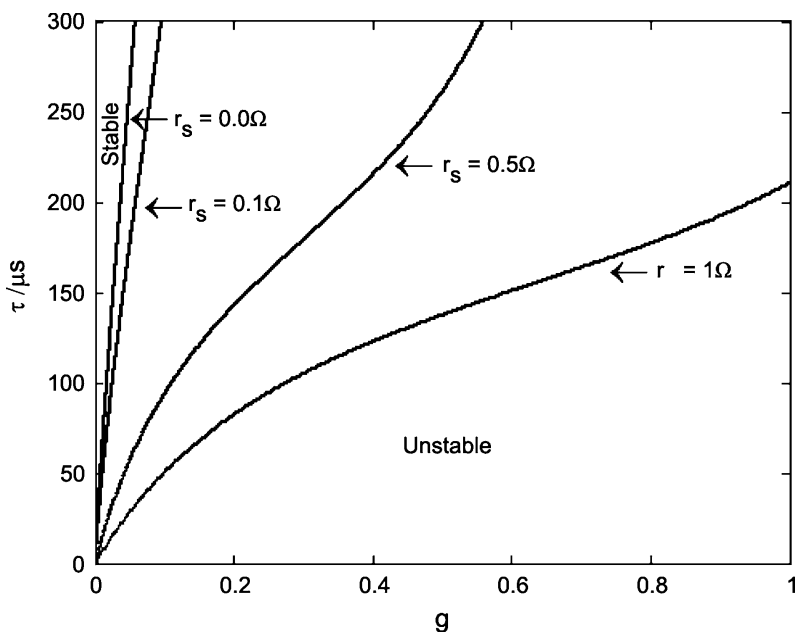

Fig. 9. Stability area in the parameter space $(g, \tau)$ from the averaged model for different values of $r_{S} . V_{L}=0$, and $V_{U}=1.5 \mathrm{~V}$. The other parameters are shown in Table I.

In order to simplify the analysis, we consider equal gain $\left(g_{P A}=\right.$ $\left.g_{N B}=g\right)$ and equal time constants $\left(\tau_{P}=\tau_{N}=\tau\right)$ for the PI controller. Fig. 9 shows the boundary between the stable and the unstable regions in the $(g, \tau)$ parameter space for different values of the current-sensor gain.

It should be noted that the validity of the results obtained by an averaged model depends greatly on the assumptions used to obtain this model. First, a high switching frequency should be used, which implies a small ripple of the state variables. In addition, the feedback coefficients should ensure that the control voltages lie between the upper and lower bounds of the ramp voltage. If these assumptions are not met, the system may have subharmonic instability or an NS bifurcation even if the average model predicts a stable behavior.

In the next section, some instability phenomena that can occur in the system are presented, and it is shown that some of these are not detected by the averaged model.

\section{Different Instability Phenomena From the SWITCHED MODEL}

Numerical simulations with the switched model were performed to determine the possible bifurcation phenomena that the circuit can exhibit. In the following subsections, two different instability phenomena are obtained by varying the proportional gain of the voltage controllers, the current sensor gain, and the amplitude of the ramp voltage. Similar behavior can be obtained by varying other parameters. The fixed parameters used are those shown in Table I. The rest of the parameters are shown in the figure captions.

\section{A. PD Bifurcation and Subharmonic Oscillations}

If the current sensor gain $r_{S}$ and the feedback coefficient $g$ are relatively large, the system has a PD bifurcation if the amplitude $V_{U}$ of the ramp signal is decreased. Fig. 10 shows the inductor current with a 2T-periodic waveform after a PD bifurcation [36], [37].

\section{B. NS Bifurcation and Low-Frequency Oscillations}

For relatively low values of the feedback gains $r_{S}$ and $g$, the system has low-frequency oscillations if the amplitude of the

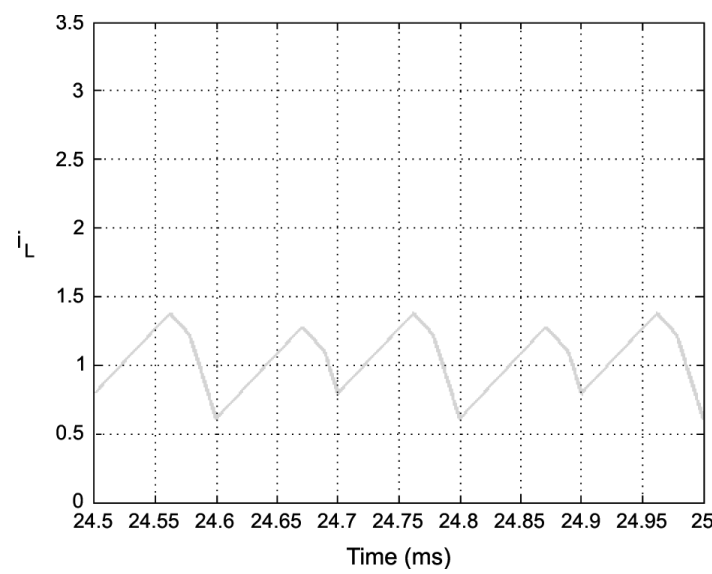

Fig. 10. Numerical simulation waveforms of the steady-state behavior of the TITO dc-dc converter after it has undergone a PD bifurcation. $g=0.05, V_{L}=$ $0 \mathrm{~V}, V_{U}=1.08 \mathrm{~V}, \tau=250 \mu \mathrm{s}$, and $r_{S}=1 \Omega$ (the other parameters are shown in Table I).

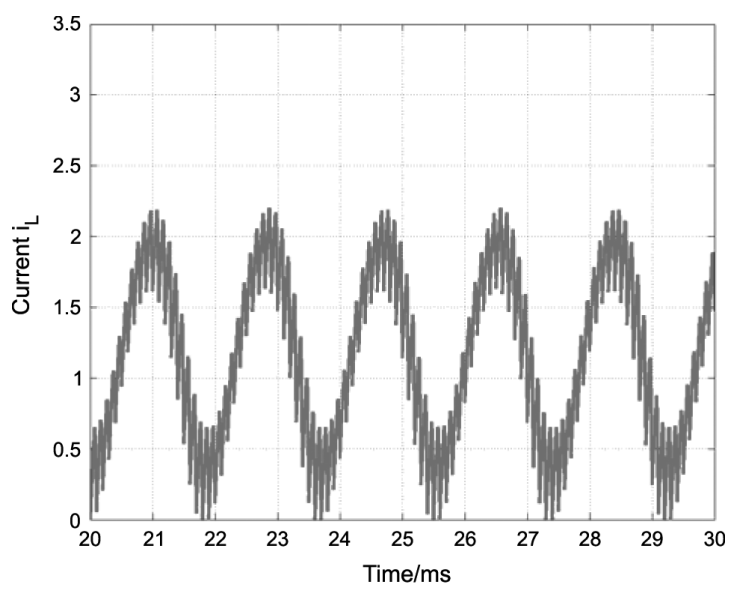

Measure date:09/11/2007

CH1: .200V/DIV DC TB A:1 ms TR:CH1+AC PT:75

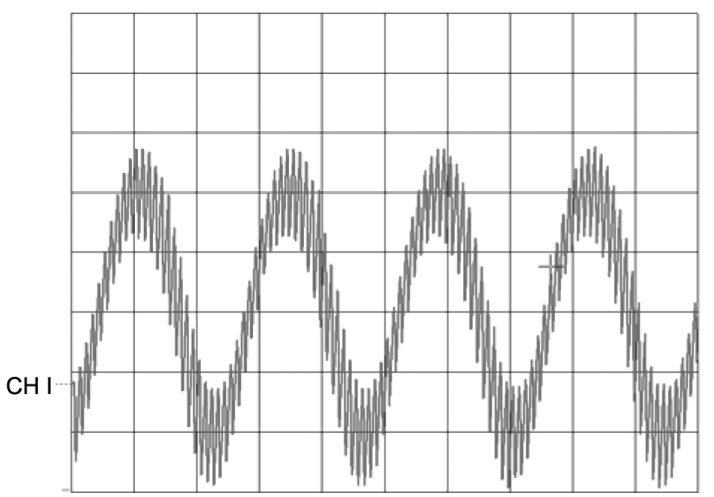

Fig. 11. Waveforms of the steady-state behavior of the TITO dc-dc converter after undergoing an NS bifurcation: (top) numerical simulations and (bottom) experimental measurements. $g=0.05, V_{L}=-1 \mathrm{~V}, V_{U}=0.5 \mathrm{~V}, \tau=$ $250 \mu \mathrm{s}$, and $r_{S}=0.085 \Omega$. The other parameter values are shown in Table I.

ramp signal is decreased. These slow oscillations are due to a Neimark-Sacker (NS) bifurcation, also known as the secondary Hopf bifurcation, which causes the birth of an invariant torus or quasi-periodic instability from a stable limit cycle [36]-[38]. Note that the NS bifurcation occurs in the Poincare section of a limit cycle of the continuous-time switched system. Fig. 11 shows the waveforms of the inductor current after this bifurcation occurs in the system. 
After NS bifurcation takes place, the system operates intermittently between CCM and DCM. It was observed in the numerical simulations that the last operating mode is required for the resulting torus to be stable. Under exclusive CCM mode, the quasi-periodic behavior is unstable or has a very small basin of attraction.

\section{THE Discrete-TIME MODEL OF THE TITO CONVERTER}

Elementary dc-dc converters, such as the buck under PWM voltage-mode control or the boost under current-programmed control, are prone to subharmonic oscillations that cannot be understood using the averaged model [5]-[12].

Recently, it has been shown that for single-switched dc-dc converters, the NS bifurcation can be detected by using a simple averaged model if the expression of the duty cycle is obtained by assuming that the control voltage is constant during one switching cycle [21], [22]. For our system, numerical simulations show that using this assumption makes the corresponding closed-loop averaged model unable to predict this bifurcation for very small values of the feedback voltage gains. However, by assuming the control voltage to be piecewise linear with different slopes during each corresponding subinterval, the model is enhanced in such a way that this phenomenon is detected more accurately. Nevertheless, the averaged model still fails to predict PD bifurcation even with this improvement. The usual tool for studying the stability of the limit cycles in switching converters and predicting most of the possible instabilities is the discrete-time model. This approach has been applied successfully to explain the nonlinear and complex behavior that can occur in elementary and paralleled dc-dc power electronic circuits [39].

\section{A. Expression of the Discrete-Time Model}

The discrete-time model can be defined as

$$
\mathbf{P}: \Sigma_{0} \rightarrow \Sigma_{0} \quad \text { and } \quad \mathbf{x}_{n} \rightarrow \mathbf{x}_{n+1}:=\mathbf{P}\left(\mathbf{d}, \mathbf{x}_{n}\right)
$$

where $\mathbf{d}=\left(d_{A}, d_{B}\right)^{T}$ is the vector of the duty cycles and $\Sigma_{0}=$ $\{x(t) / t=n T, n \in \mathbb{Z}\}$ is a suitable section in the state space. This model is useful because its fixed points $X^{*}$ correspond to periodic orbits $x^{*}$ of the continuous-time switched system, and the stability properties are the same for both of them. The expression of map $\mathbf{P}$ can be written in the following form:

$$
\mathbf{P}\left(\mathrm{d}, \mathbf{x}_{n}\right)=\boldsymbol{\Phi}(\mathrm{d}) \mathbf{x}_{n}+\Psi(\mathrm{d})
$$

where

$$
\begin{aligned}
& \boldsymbol{\Phi}(\mathbf{d})=\phi_{3}\left(\delta_{3}\right) \phi_{2}\left(\delta_{2}\right) \phi_{1}\left(\delta_{1}\right) \\
& \mathbf{\Psi}(\mathbf{d})=\phi_{3}\left(\delta_{3}\right) \phi_{2}\left(\delta_{2}\right) \psi_{1}\left(\delta_{1}\right)+\phi_{3}\left(\delta_{3}\right) \psi_{2}\left(\delta_{2}\right)+\psi_{3}\left(\delta_{3}\right)
\end{aligned}
$$

in which it is considered that the solution of $\dot{\mathbf{x}}=\mathbf{A}_{k} \mathbf{x}+\mathbf{B}_{k}$, with initial condition $\mathbf{x}_{0}$ at time instant $t_{0}$, can be written as $\mathbf{x}(t)=\phi_{k}\left(t-t_{0}\right) \mathbf{x}_{0}+\psi_{k}\left(t-t_{0}\right)$, and that $\delta_{k}$ is the duration of phase $k: \delta_{1}=\min \left(d_{A}, d_{B}\right), \delta_{2}=\left|d_{A}-d_{B}\right|$, and $\delta_{3}=$ $1-\left(\delta_{1}+\delta_{2}\right)$. A fixed point of $\mathbf{P}$ is a point $\mathbf{X}^{*}$ in the state space for which we have $\mathbf{X}^{*}=\mathbf{P}\left(\mathbf{X}^{*}, \mathbf{d}^{*}\right)$. This fixed point can be obtained by enforcing periodicity in the expression of map $\mathbf{P}$.

\section{B. Jacobian Matrix of the Discrete-Time Model}

The stability analysis of the nominal periodic orbit of the closed-loop system, represented by $\mathbf{X}^{*}$, can be carried out by using the eigenvalues of the Jacobian matrix DPevaluated at the fixed point $\mathbf{X}^{*}$. This can be done by solving the characteristic equation for the fixed point $\mathbf{X}^{*}$, which is given by

$$
\operatorname{det}(\mathbf{D P}-\lambda \mathbf{I})=0 .
$$

A well-known sufficient condition for instability is that one of the eigenvalues has a modulus greater than one.

At a certain bifurcation point, the modulus of one (real) eigenvalue $\lambda$ or two (complex conjugates) eigenvalues $\lambda_{1}$ and $\lambda_{2}$ is equal to one, and, generally, we can write $\lambda=e^{\mathrm{j} \theta}$. There are three different standard ways for an eigenvalue to leave the unit circle in the case of the smooth map $\mathbf{P}$. In the case of a real eigenvalue, there are two different cases: For $\theta=\pi(\lambda=$ $-1)$, a PD bifurcation takes place, while for $\theta=0(\lambda=1)$, saddle-node bifurcation can occur in which a pair of periodic orbits coalesces and disappears [36], [37]. For other values of $\theta$, the eigenvalues will be complex conjugates, and, regardless of where they cross the unit circle, an NS bifurcation is possible.

In [40], it is shown that for single switched converters, the Jacobian matrix expression can be written as follows:

$$
\mathbf{D P}=\boldsymbol{\Phi}(\mathbf{d})-\frac{\partial \mathbf{P}}{\partial \mathbf{d}}\left(\frac{\partial \boldsymbol{\sigma}}{\partial \mathbf{d}}\right)^{-1}\left(\frac{\partial \boldsymbol{\sigma}}{\partial \mathbf{x}_{n}}\right)
$$

where $\sigma$ is a function obtained from the switching condition that relates the switching time (or duty cycle) implicitly to the state variables. It can be shown that the same expression (22) applies for multiple switches and multiple configuration converters, where $\sigma$ is a vector function relating the switching times (or duty cycles) to the state variables [27]. In our case, $\sigma$ is the column vector given by

$$
\boldsymbol{\sigma}(\mathbf{d}, \mathbf{x})=\left(\begin{array}{c}
\sigma_{B}\left(d_{B}, \mathbf{x}\right) \\
\sigma_{A}\left(d_{A}, \mathbf{x}\right.
\end{array}\right) .
$$

The partial derivatives with respect to $\mathbf{x}_{\mathbf{n}}$ and $\mathbf{d}$ can be obtained by (24) and (25) shown at the bottom of the page, where

$$
\begin{aligned}
\Delta \dot{\mathbf{x}}_{k} & =\dot{\mathbf{x}}_{k}^{-}-\dot{\mathbf{x}}_{k}^{+} \\
\dot{\mathbf{x}}_{1}^{-} & =\mathbf{A}_{1}\left(\phi_{1}\left(\delta_{1}\right) \mathbf{x}_{n}+\psi_{1}\left(\delta_{1}\right)\right)+\mathbf{B}_{1} \\
\dot{\mathbf{x}}_{1}^{+} & =\mathbf{A}_{2}\left(\phi_{1}\left(\delta_{1}\right) \mathbf{x}_{n}+\psi_{1}\left(\delta_{1}\right)\right)+\mathbf{B}_{2}
\end{aligned}
$$

$$
\begin{aligned}
& \frac{\partial \boldsymbol{\sigma}\left(\mathbf{d}, \mathbf{x}_{n}\right)}{\partial \mathbf{x}_{n}}=-\left(\begin{array}{c}
G_{X B} \phi_{1}\left(\delta_{1}\right) \\
G_{X A} \phi_{2}\left(\delta_{2}\right) \phi_{1}\left(\delta_{1}\right)
\end{array}\right) \\
& \frac{\partial \boldsymbol{\sigma}\left(\mathbf{d}, \mathbf{x}_{n}\right)}{\partial \mathbf{d}}=\left(\begin{array}{cc}
-T G_{X B} \dot{\mathbf{x}}_{1}^{-}+V_{U}-V_{L} & 0 \\
-T G_{X A} \phi_{2} \Delta \dot{\mathbf{x}}_{1} & -T G_{X A} \phi_{2} \dot{\mathbf{x}}_{2}^{+}+V_{U}-V_{L}
\end{array}\right)
\end{aligned}
$$




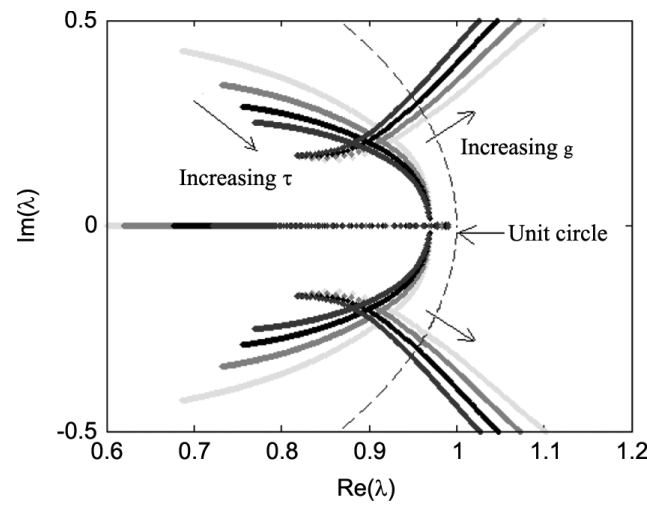

(a)

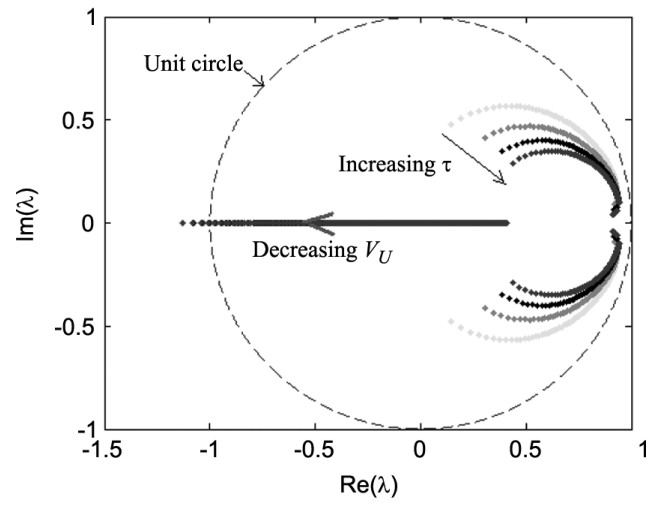

(b)

Fig. 12. Evolution of the eigenvalues of the discrete-time model for different values of the time constant $\tau$ when (a) $g$ is increased and an NS bifurcation occurs $\left(V_{L}=0 \mathrm{~V}, V_{U}=1.5 \mathrm{~V}\right.$, and $\left.r_{S}=0.085 \Omega\right)$ and $(\mathrm{b})$ when $V_{U}$ is decreased and a PD bifurcation takes place $\left(V_{L}=0 \mathrm{~V}, g=0.05\right.$, and $r_{S}=1 \Omega$ ). The other parameter values are shown in Table I.

$$
\begin{aligned}
& \dot{\mathbf{x}}_{2}^{-}=\mathbf{A}_{2}\left(\phi_{2}\left(\delta_{2}\right) \phi_{1}\left(\delta_{1}\right) \mathbf{x}_{n}+\phi_{2}\left(\delta_{2}\right) \psi_{1}\left(\delta_{1}\right)+\psi_{2}\left(\delta_{2}\right)\right)+\mathbf{B}_{2} \\
& \dot{\mathbf{x}}_{2}^{+}=\mathbf{A}_{3}\left(\phi_{2}\left(\delta_{2}\right) \phi_{1}\left(\delta_{1}\right) \mathbf{x}_{n}+\phi_{2}\left(\delta_{2}\right) \psi_{1}\left(\delta_{1}\right)+\psi_{2}\left(\delta_{2}\right)\right)+\mathbf{B}_{3} .
\end{aligned}
$$

Since the vector field is discontinuous, the state-variable derivatives $\dot{\mathrm{x}}_{k}^{-}$just before switching are different from the derivatives $\dot{\mathbf{x}}_{k}^{+}$just after switching. The partial derivative of the map function $\mathbf{P}$ with respect to $\mathbf{d}$ can be obtained from (18) in the same way, i.e.,

$$
\frac{\partial \mathbf{P}}{\partial \mathbf{d}}=T\left(\phi_{3}\left(\delta_{3}\right) \phi_{2}\left(\delta_{2}\right) \Delta \dot{\mathbf{x}}_{1} \quad \phi_{3}\left(\delta_{3}\right) \Delta \dot{\mathbf{x}}_{2}\right)
$$

\section{Stability Boundaries From the Discrete-Time Model}

The eigenvalues of the discrete-time Jacobian matrix are used to obtain the stability region in certain suitable design parameter planes, for example, $(g, \tau)$ and $\left(V_{U}, \tau\right)$. In both cases, for the four values of $\tau$ within the range $(100,250) \mu$ s, we increase $g$ in the interval $(0.01,0.2)$ or decrease $V_{U}$ from 1.5 down to 1 $\mathrm{V}$. The eigenvalues of the discrete-time Jacobian matrix, as $g$ is increased or $V_{U}$ decreased, are shown in Fig. 12.

It can be seen that there are two different mechanisms for losing stability. When $V_{U}$ decreases, a PD bifurcation takes place. If $g$ is increased, an NS bifurcation occurs. These results can be used to obtain the boundary between stable and unstable regions in appropriate parameter spaces. The stability boundary

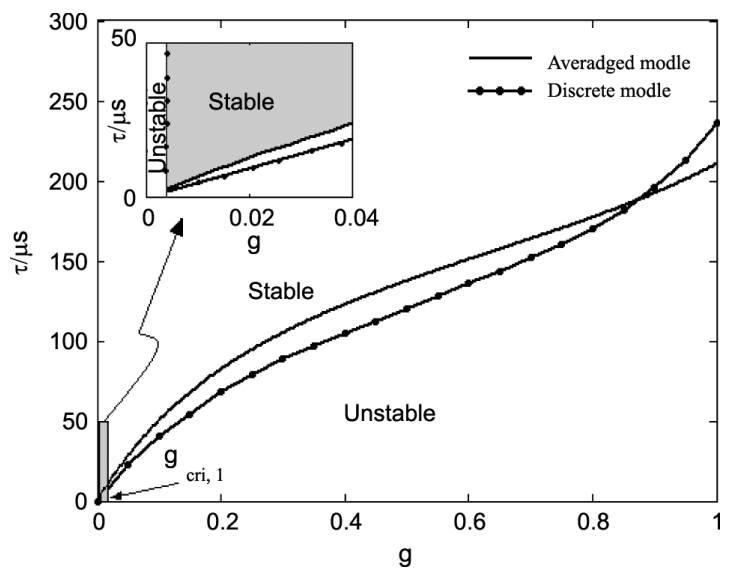

Fig. 13. Stability area in the parameter space $(g, \tau)$ from the discrete-time model and the averaged model. $r_{S}=1 \Omega, V_{L}=0$, and $V_{U}=1.5 \mathrm{~V}$. The other parameter values are shown in Table I.

in the parameter space $(g, \tau)$ is shown in Fig. 13 from the largest absolute value of the eigenvalues of the Jacobian matrix. In the same figure, the result of Fig. 9, obtained from the averaged model for the case of $r_{S}=1 \Omega$, is also shown. There are some discrepancies between these results because the averaged model does not predict the bifurcation points accurately. Moreover, the stability boundary corresponding to the discrete-time model corresponds to a PD or an NS bifurcation, while the boundary obtained from the averaged model corresponds only to a Hopf bifurcation. For feedback gains $g$ higher than a critical value $g_{\mathrm{cri}, 1}$, which is independent of the time constant $\tau$, all the complex eigenvalues are inside the unit circle. By increasing $g$ and for another critical value $g_{\mathrm{cri}, 2}$, two complex conjugate eigenvalues cross the unit circle, which indicates an NS bifurcation. The critical value $g_{\text {cri,2 }}$ depends on the time constant of the PI controller $\tau$ and on other parameters, such as the amplitude of the ramp voltage $V_{U}$.

Furthermore, the eigenvalues of the discrete-time model show that for lower values of the gain coefficients and the time constant of the integrators, the subharmonic oscillations in the discrete-time model occur after a Hopf bifurcation in the averaged model. However, this is not so for relatively large values of these parameters, in which case, the discrete-time model predicts an NS bifurcation, and the averaged model predicts a Hopf bifurcation, but the critical values of the parameters for which bifurcations occur are slightly different.

The actual stability region is different from the predicted region using the averaged model even when PD instability does not occur, which happens for relatively small values of the feedback gain. A small rectangular area, which is enlarged in Fig. 13, contains an NS bifurcation from the discrete-time and the switched models, and stable behavior is predicted by the averaged model. This figure shows that below $g_{\text {cri, } 1}$, the system has a low-frequency instability.

\section{NONSMOOTH BIFURCATIONS}

The discrete-time model defined earlier is restricted to the switching pattern that is specific for the normal mode of operation $\left(M_{1}\right.$ or $\left.\boldsymbol{M}_{2}\right)$. However, in certain parameter ranges, this pattern can be modified, mainly for subharmonic oscillations or chaotic regimes. Therefore, it is important to establish the limits 
of the model, which are related to the following boundary conditions.

1) The appearance of DCM: Although, in general, we found persistence after this bifurcation occurred, a specific discrete model that includes a fourth configuration (open inductor) should be analyzed in order to determine the stability and find other possible scenarios, such as nonsmooth flip bifurcation. In the discrete-time model, the boundary of this border-collision bifurcation is obtained by adding the following conditions to the fixed-point conditions (see Fig. 14(a)):

$$
i_{L}(t)=0 \text {. }
$$

2) The suppression of the series configuration $\left(\mathcal{C}_{4}\right)$, which is the last one of every period in the sequence of the main mode, either $\boldsymbol{M}_{1}$ or $\boldsymbol{M}_{2}$ : This bifurcation, which will be denoted as SCS, is a particular case for this kind of circuit and control and occurs if the intermediate configuration $\left(\mathcal{C}_{2}\right.$ or $\mathcal{C}_{3}$ ) is maintained until the end of the cycle; thus, the development of $\mathcal{C}_{4}$ is inhibited [see Fig. 14(b)], and, consequently, the charging process of the capacitor of one of the outputs does not take place. Under these conditions, the dynamics of the system becomes very noisy because the action of the integral control forces the modulating signals to alternate their positions in order to allow from time to time an abrupt load of the capacitor filter. Let us assume, without loss of generality, that the circuit operates in mode $\boldsymbol{M}_{1}\left(d_{A}>d_{B}\right)$, and the parameters defining the loads are progressively changed in such a way that mode $M_{2}\left(d_{A}>d_{B}\right)$ is forced. If the slope of the modulating ramp is high enough to enable the second asynchronous switching, there will be a continuous transition from $M_{1}$ to $M_{2}$ through the sequence $\mathcal{C}_{1} \rightarrow \mathcal{C}_{4}\left(d_{A}=d_{B}\right)$. However, if the inductor current decreases faster, then the second asynchronous switching (and therefore $\mathcal{C}_{4}$ ) will be skipped. The two possible boundaries for this bifurcation can be approximated by

$$
\begin{aligned}
& \mathcal{C}_{2} \text { holds : } \frac{V_{U}-V_{L}}{r_{S} T}+\frac{V_{I N}-V_{P}}{L}=0 \\
& \mathcal{C}_{3} \text { holds : } \frac{V_{U}-V_{L}}{r_{S} T}+\frac{V_{N}}{L}=0
\end{aligned}
$$

3) The saturation of duty cycles: Apart from the aforementioned bifurcations concerning the main operation modes, saturation of one of the duty cycles is indeed possible. In addition, considering $M_{1}$ as the main mode, a first possible boundary corresponds to $d_{A}=1$ [from $M_{1}$ to the $\mathcal{C}_{1} \rightarrow \mathcal{C}_{2}$ sequence, see Fig. 14(c)]. Beyond this, a second possible boundary is associated with $d_{B}=1$ [from the $\mathcal{C}_{1} \rightarrow \mathcal{C}_{2}$ sequence to the single configuration $\mathcal{C}_{1}$, see Fig. 14(d)]. Otherwise, considering $M_{2}$, similar cases would correspond to $d_{B}=1$ (from $M_{1}$ to $\mathcal{C}_{1} \rightarrow \mathcal{C}_{3}$ ) followed by $d_{A}=1$ (from $\mathcal{C}_{1} \rightarrow \mathcal{C}_{3}$ to $\mathcal{C}_{1}$ ). However, these bifurcations will be present only for $n T$-periodic limit cycles, when $n>1$, and the aforementioned conditions apply only for one period of the modulating signal among those $n$ periods. As for DCM, the resulting dynamics after these bifurcations should be (a)

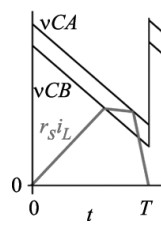

(b)

(c)

(d)

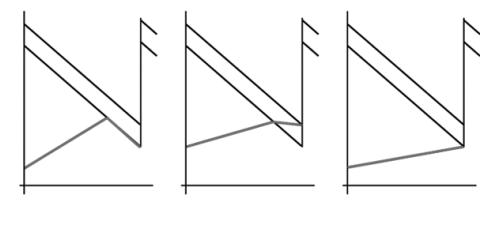

Fig. 14. Illustrative figure for different nonsmooth bifurcations: (a) DCM, (b) SCS, and duty-cycle saturations for the (c) first and (d) second occurrence.

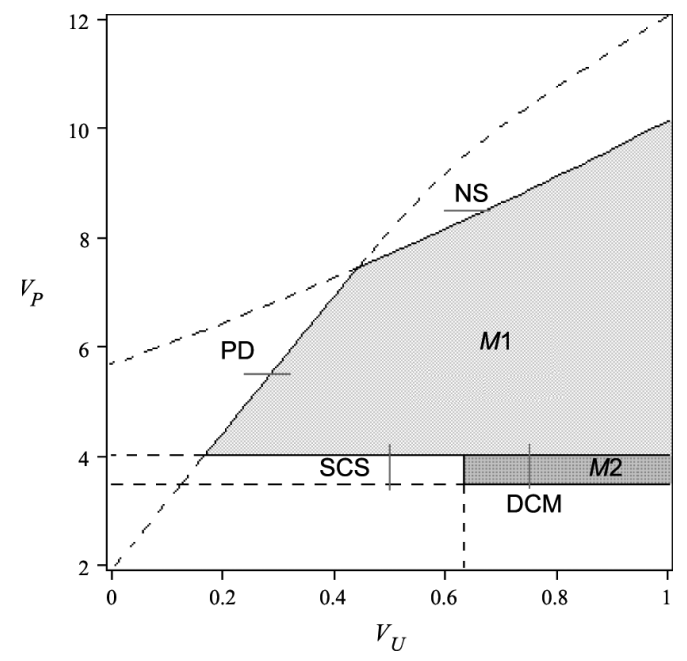

Fig. 15. Bifurcation diagram in $\left(V_{U}, V_{P}\right)$ parameter subspace. Regions for $1 T$-periodic stable orbits, $\boldsymbol{M}_{1}$ and $\boldsymbol{M}_{2}$, are highlighted. Boundaries for NS, flip (PD), SCS, and DCM bifurcations are plotted.

investigated by considering the existence and stability of the modified sequences of configurations in the limit cycle.

In Fig. 15, the region in a $\left(V_{U}, V_{P}\right)$ parameter subspace for $1 T$-periodic behavior has been highlighted, including the two main modes $M_{1}$ and $M_{2}$. Their boundaries are obtained by the following conditions applied to an approximation of the discrete-time model. The NS curve corresponds to a complex conjugate eigenvalue pair with unit modulus, whereas the flip or PD curve corresponds to an eigenvalue equal to -1 . In this second case, the duty-cycle saturation in the $2 T$-periodic orbit plays a relevant role. The suppression of the SCS curve is plotted using (29), and the DCM is given by (28).

Some 1-D bifurcation diagrams, which have been made following the red segments that intersect the aforementioned boundaries (see Fig. 16), are shown in Fig. 16. Note that in the DCM diagram (bottom right), the previous transition from $M_{1}$ to $M_{2}$ cannot be appreciated, and the final result is just the persistence phenomena in the DCM bifurcation. However, in the SCS diagram (bottom left), mode $M_{2}$ cannot be attained because the value of the parameter $V_{U}$ is lower than its existence limit (30); thus, there is a transition to high-order subharmonics and chaos characteristic of maps with discontinuities.

\section{CONCLUSION}

A single-inductor TITO switching dc-dc converter has been analyzed with both static and dynamic system properties. This converter, which does not need symmetry in its positive and negative outputs, operates with a PWM control including two 

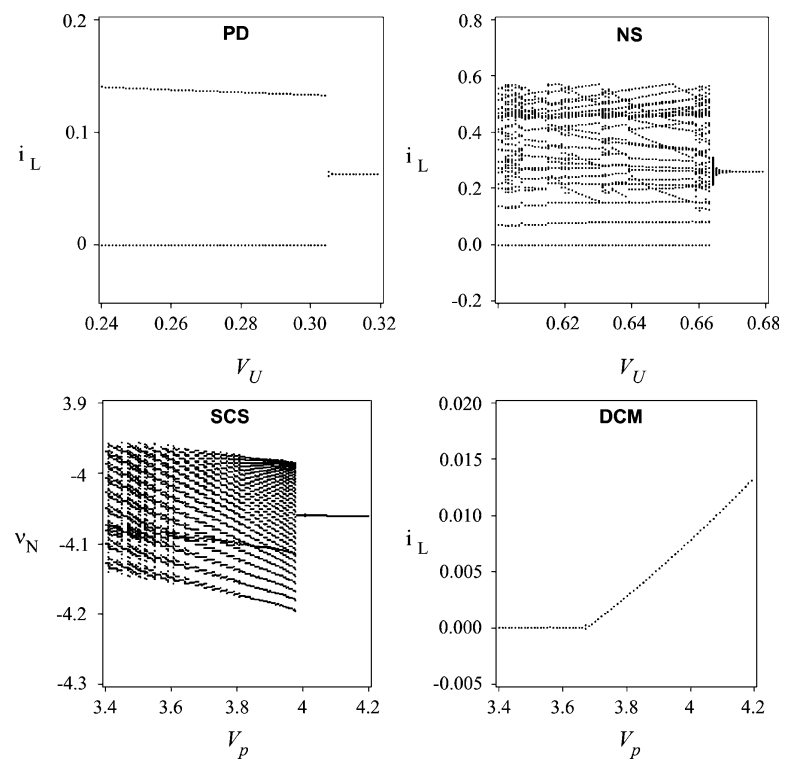

Fig. 16. One-dimensional diagrams, which follow the red segments in this figure, showing the different bifurcation schemes: PD $\left(V_{P}=5.5 \mathrm{~V}\right)$, NS $\left(V_{P}=8.5 \mathrm{~V}\right)$, SCS $\left(V_{U}=5.0 \mathrm{~V}\right)$, and $\mathrm{DCM}\left(V_{U}=7.5 \mathrm{~V}\right)$. The varying parameter is in the abscissa, and the synchronous value for one selected phase is in the ordinate.

PI blocks, whose inputs are the error signals. This control has proven capable of providing stable behavior if the parameters are properly selected. The averaging approach has been used to make the analysis more comprehensive, including details of the steady-state properties and an explicit expression for the Jacobian matrix in order to check stability. However, this model can fail to predict some stability boundaries, so a discrete-time model is necessary in cases such as PD, and sometimes, even NS bifurcations. In general, the averaged model and direct simulations from the switched model are similar enough in cases not involving fast-scale (switching) instabilities due to the presence of excessive ripple in the control voltages. Experimental measurements have been used to confirm the analytical and simulation results. Finally, some bifurcation diagrams have been selected to illustrate different boundaries of the normal operation regime, including the existence and stability conditions. A detailed study of these nonsmooth bifurcations, which are due to discontinuities in the power stage or in the control, will be the subject of future research.

\section{ACKNOWLEDGMENT}

The authors would like to thank the anonymous reviewers for their valuable comments and suggestions that helped improve an original manuscript of this work.

\section{REFERENCES}

[1] R. Giral, J. Calvente, R. Leyva, A. El Aroudi, G. Arsov, and L. Martinez-Salamero, "Symmetrical power supply for 42-V automotive applications," Facta Univ. (NIS), Electron. Energ., vol. 17, pp. 363-374, 2004.

[2] Y. Xi and P. K. Jain, "A forward converter topology with independent and precisely regulated multiple outputs," IEEE Trans. Power Electron., vol. 18, no. 2, pp. 648-658, Mar. 2003.

[3] R. Barabás, B. Buti, J. Hamar, and I. Nagy, "Control characteristics, simulation and test results of a dual channel DC-DC converter family," in Proc. SPEEDAM, Capri, Italy, 2004.
[4] L. Benadero, R. Giral, A. El Aroudi, and J. Calvente, "Stability analysis of a single inductor dual switching DC-DC converter," Math. Comput. Simul., vol. 71, no. 4-6, pp. 256-269, 2006.

[5] C. K. Tse and M. Di Bernardo, "Complex behavior in switching power converters," Proc. IEEE, vol. 90, no. 5, pp. 768-781, May 2002.

[6] M. di Bernardo and F. Vasca, "Discrete-time maps for the analysis of bifurcations and chaos in DC/DC converters," IEEE Trans. Circuits Syst. I, Reg. Papers, vol. 47, no. 2, pp. 130-143, Feb. 2000.

[7] J. H. B. Deane and D. C. Hamill, "Instability, sub-harmonics and chaos in power electronic systems," IEEE Trans. Power Electron., vol. 5, no. 3, pp. 260-268, Jul. 1990.

[8] A. El Aroudi, M. Debbat, G. Olivar, L. Benadero, E. Toribio, and R. Giral, "Bifurcations in DC-DC switching converters review of methods and applications," Int. J. Bifur. Chaos, vol. 15, no. 5, pp. 1549-1578, May 2005.

[9] C. K. Tse, Complex Behavior of Switching Power Converters. Boca Raton, FL: CRC Press, 2004.

[10] B. Robert and C. Robert, "Border collision bifurcations in a chaotic PWM H-bridge single-phase inverter," in Proc. 10th EPE-PEMC, 2002.

[11] S. Banerjee, E. Ott, J. A. Yorke, and G. H. Yuan, "Anomalous bifurcations in DC-DC converters: Borderline collisions in piecewise smooth maps," in Proc. IEEE PESC, 1997, vol. 2, pp. 1337-1344.

[12] Z. T. Zhusubaliyev and E. Mosekilde, Bifurcation and Chaos in Piecewise-Smooth Systems. , Singapore: World Scientific, 2003.

[13] S. Maity, D. Tripathy, T. K. Bhattacharya, and S. Banerjee, "Bifurcation analysis of PWM-1 voltage-mode-controlled buck converter using the exact discrete model," IEEE Trans. Circuits Syst. I, Reg. Papers, vol. 54, no. 5, pp. 1120-1130, May 2007.

[14] F. Angulo, G. Olivar, and M. di Bernardo, "Two-parameter discontinuity-induced bifurcation curves in a ZAD-strategy-controlled DC-DC buck converter," IEEE Trans. Circuits Syst. I, Reg. Papers, vol. 55, no. 8, pp. 2392-2401, Sep. 2008.

[15] B. K. H. Wong, H. S. H. Chung, and S. T. S. Lee, "Computation of the cycle state-variable sensitivity matrix of PWM DC/DC converters and its applications," IEEE Trans. Circuits Syst. I, Reg. Papers, vol. 47, no. 10, pp. 1542-1548, Oct. 2000.

[16] I. A. Hiskens and M. A. Pai, "Trajectory sensitivity analysis of hybrid systems," IEEE Trans. Circuits Syst. I, Reg. Papers, vol. 47, no. 2, pp. 204-220, Feb. 2000.

[17] O. Dranga, B. Buti, I. Nagy, and H. Funato, "Stability analysis of nonlinear power electronic systems utilizing periodicity and introducing auxiliary state vector," IEEE Trans. Circuits Syst. I, Reg. Papers, vol. 52, no. 1, pp. 168-178, Jan. 2005.

[18] A. Elbkosh, D. Giaouris, V. Pickert, B. Zahawi, and S. Banerjee, "Stability analysis and control of bifurcations of parallel connected DC/DC converters using the monodromy matrix," in Proc. IEEE ISCAS, May 18-21, 2008, pp. 556-559.

[19] D. Giaouris, S. Banerjee, B. Zahawi, and V. Pickert, "Stability analysis of the continuous-conduction-mode buck converter via Filippov's method," IEEE Trans. Circuits Syst. I, Reg. Papers, vol. 55, no. 4, pp. 1084-1096, May 2008.

[20] D. Giaouris, S. Banerjee, B. Zahawi, and V. Pickert, "Control of fast scale Bifurcations in power-factor correction converters," IEEE Trans. Circuits Syst. II, Exp. Briefs, vol. 54, no. 9, pp. 805-809, Sep. 2007.

[21] C. K. Tse, Y. M. Lai, and H. H. C. Iu, "Hopf bifurcation and chaos in a free-running current-controlled Cuk switching regulator," IEEE Trans. Circuits Syst. I, Reg. Papers, vol. 47, no. 4, pp. 448-457, Apr. 2000.

[22] S. C. Wong, X. Wu, and C. K. Tse, "Sustained slow-scale oscillation in higher order current-mode controlled converter," IEEE Trans. Circuits Syst. II, Exp. Briefs, vol. 55, no. 5, pp. 489-493, May 2008.

[23] A. El Aroudi, M. Orabi, and L. Martinez-Salamero, "A representative discrete-time model for uncovering slow and fast scale instabilities in boost power factor correction AC-DC pre-regulators," Int. J. Bifur. Chaos., vol. 18, no. 10, pp. 3073-3092, 2008.

[24] M. Orabi and T. Ninomiya, "Nonlinear dynamics of power-factor-correction converter," IEEE Trans. Ind. Electron., vol. 50, no. 6, pp. 1116-1125, Dec. 2003.

[25] S. C. Wong, C. K. Tse, M. Orabi, and T. Ninomiya, "The method of double averaging: An approach for modeling power-factor-correction power converters," IEEE Trans. Circuits Syst. I, Reg. Papers, vol. 53, no. 2, pp. 454-462, Feb. 2006

[26] X. Wu, C. K. Tse, O. Dranga, and J. Lu, "Fast-scale instability of singlestage power-factor-correction power supplies," IEEE Trans. Circuits Syst. I, Reg. Papers, vol. 53, no. 1, pp. 204-213, Jan. 2006. 
[27] A. El Aroudi, M. Debbat, and L. Martinez-Salamero, "Poincaré maps modelling and local orbital stability analysis of discontinuous piecewise affine periodically driven systems," Nonlinear Dynamics, vol. 50, no. 3, pp. 431-445, Nov. 2007.

[28] A. El Aroudi, B. G. M. Robert, A. Cid-Pastor, and L. Martinez-Salamero, "Modelling and design rules of a two-cell buck converter under a digital controller," IEEE Trans. Power Electron., vol. 23, no. 2, pp. 859-870, Mar. 2008.

[29] I. Nagy and O. Dranga, "Bifurcation in a dual channel resonant DC-DC converter," in Proc. IEEE ISIE, 2000, pp. 495-500.

[30] B. Buti, O. Dranga, H. Funato, and I. Nagy, "Stability analysis of nonlinear power electronic systems utilizing periodicity and introducing auxiliary state vector," IEEE Trans. Circuits Syst. I, Reg. Papers, vol. 52, no. 1, pp. 168-178, Jan. 2005.

[31] O. Dranga, B. Buti, and I. Nagy, "Stability analysis of a feedback-controlled resonant DC-DC converter," IEEE Trans. Ind. Electron., vol. 50, no. 1, pp. 141-152, Feb. 2003.

[32] W. H. Ki and D. Ma, "Single-inductor multiple-output switching converters," in Proc. IEEE PESC, 2001, vol. 1, pp. 226-231.

[33] D. Ma, W. H. Ki, C. Y. Tsui, and P. K. T. Mok, "Single-inductor multiple-output switching converters with time-multiplexing control in discontinuous conduction mode," IEEE J. Solid-State Circuits, vol. 38, no. 1, pp. 89-100, Jan. 2003.

[34] D. Ma, W. H. Ki, and C. Y. Tsui, "A pseudo-CCM/DCM SIMO switching converter with freewheel switching," IEEE J. Solid-State Circuits, vol. 38, no. 6, pp. 1007-1014, Jun. 2003.

[35] D. Trevisan, W. Stefanutti, P. Mattavelli, and P. Tenti, "FPGA control of SIMO DC-DC converters using load current estimation," in Proc. 32nd IEEE IECON, 2005, pp. 2243-2248.

[36] Y. A. Kuznetsov, "Elements of applied bifurcation theory," in Applied Mathematical Sciences. New York: Springer-Verlag, 1998, vol. 112.

[37] J. Guckenheimer and P. Holmes, "Nonlinear oscillations, dynamical systems and bifurcations of vector fields," in Applied Mathematical Sciences. New York: Springer-Verlag, 1998, vol. 42.

[38] A. El Aroudi and R. Leyva, "Quasi-periodic route to chaos in a PWM voltage controlled DC-DC Boost converter," IEEE Trans. Circuits Syst. I, Reg. Papers, vol. 48, no. 8, pp. 967-987, Aug. 2001.

[39] H. H. C. Iu and C. K. Tse, "Bifurcation behaviour of parallel-connected buck converters," IEEE Trans. Circuits Syst. I, Reg. Papers, vol. 48, no. 2, pp. 233-240, Feb. 2001.

[40] J. G. Kassakian, M. F. Schlecht, and G. C. Verghese, Principles of Power Electronics. Reading, MA: Addison-Wesley, 1991.

[41] M. di Bernardo, C. Budd, A. Champenys, and B. Kowalczyk, Piecewise-Smooth Dynamical Systems: Theory and Applications. New York: Springer-Verlag, 2007.

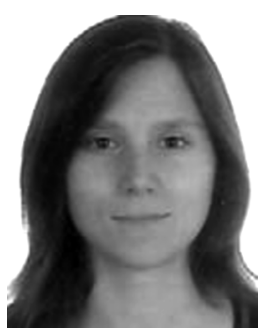

Vanessa Moreno-Font was born in Barcelona, Spain, in 1975. She received the M.Sc. degree in telecommunication engineering from the Universitat Politècnica de Catalunya (UPC), Barcelona, Spain, in 2001, where she is currently working toward the Ph.D. degree.

She has been with UPC since 2001, where she has been teaching physics and programming languages. Her research interests in nonlinear phenomena are currently focused on modeling and simulation of complex dc-dc converters.

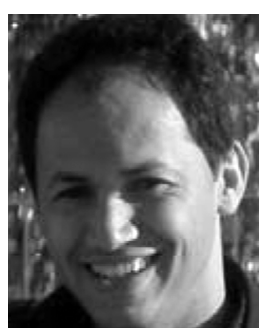

Abdelali El Aroudi (M'02) was born in Tangier, Morocco, in 1973. He received the B.S. degree in physical science from the Faculté des Sciences, Université Abdelmalek Essadi, Tetouan, Morocco, $<<\mathrm{AQ} 4>>$ in 1995 and the Ph.D. degree (with honors) from the Universitat Politècnica de Catalunya (UPC), Barcelona, Spain, in 2000.

From 1999 to 2001, he was a Visiting Professor with the Department of Electronics, Electrical Engineering and Automatic Control (DEEEA), Technical School of Engineering (ETSE), Rovira i
Virgili University (URV), Tarragona, Spain, where he became an Associate Professor in 2001 and a full-time tenure Associate Professor in 2005.<<AQ5 >> From September 2007 to January 2008, he had a visiting scholarship with the Department of Mathematics and Statistics, National University of Colombia, Manizales, Colombia, where he conducted research on the modeling of power electronic circuits for energy management. From February 2008 to July 2008, he was a visiting Scholar with the Centre de Recherche en Sciences et Technologies de l'Information et de la Communication (CReSTIC), Reims, France. He has participated in three Spanish national research projects and five cooperative international projects. He is Member of the GAEI research group (URV) in industrial electronics and automatic control, whose main research fields are power conditioning for vehicles, satellites and renewable energy. He has been invited to give talks at several universities in Europe, South America, and Africa. He has published about 90 papers in scientific journals and conference proceedings. His research interests are in the field of structure and control of power-conditioning systems for autonomous systems, power-factor correction, stability problems, nonlinear phenomena, chaotic dynamics, bifurcations, and control.

Dr. El Aroudi is a Reviewer for several scientific and technical journals.

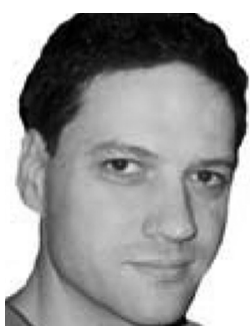

Javier Calvente (S'94-M'03) received the B.S.degree in telecommunication engineering and the Ph.D. degree from the Universitat Politècnica de Catalunya (UPC), Barcelona, Spain, in 1994 and 2001, respectively.

He was a visiting Scholar with Alcatel Space Industries, Toulouse, France, in 1998. He is currently an Associate Professor with the Department of Electronics, Electrical Engineering and Automatic Control (DEEEA), Technical School of Engineering (ETSE), Rovira i Virgili University, Tarragona, Spain, where he is working in the field of control of power converters. He is Member of the GAEI research group on Industrial Electronics and Automatic Control, whose main research fields are power conditioning for vehicles, satellites, and renewable energy.

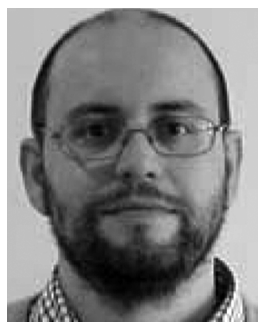

Roberto Giral (S'94-M'02) received the Ingeniero Técnico de Telecomunicación, Ingeniero de Telecomunicación, and Ph.D. (with honors) degrees from the Universitat Politècnica de Catalunya (UPC), Barcelona, Spain, in 1991, 1994, and 1999, respectively.

$\mathrm{He}$ is currently an Associate Professor with the Departamento d'Enginyeria Electrònica, Elèctrica i Automàtica, Escola Tècnica Superior d'Enginyeria (ETSE), Universitat Rovira i Virgili de Tarragona, Tarragona, Spain, where he is working in the field of power electronics. He is Member of the GAEI research group on Industrial Electronics and Automatic Control, whose main research fields are power conditioning for vehicles, satellites and renewable energy.

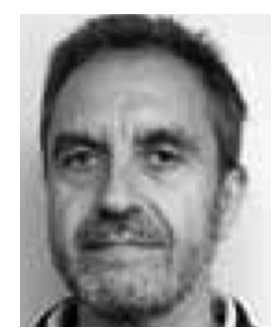

Luis Benadero was born in Ciudad Real, Spain, in 1952. He received the Ph.D. degree from the Universitat Politècnica de Catalunya (UPC), Barcelona, Spain, in 1983.

$\mathrm{He}$ has been with UPC where he was formerly with the Department of Electronic Engineering and currently an Associate Professor with the Applied Physics Department. His current research interests include nonlinear phenomena, such as bifurcations and chaotic dynamics in power electronics. 